\title{
CLASSIFICATION OF METABELIAN $p$-GROUPS
}

$\mathrm{BY}$

\author{
WU-NAN CHOU
}

\begin{abstract}
Let $G$ be a two-generator metabelian group of exponent $p$ with class of nilpotence $c$, where $c \leq p-1$ and $p$ is an odd prime. In this paper, we shall consider the classification problem when $\left|G_{2} / G_{3}\right|=p,\left|G_{3} / G_{4}\right|=p^{2}$ and $\left|G_{4} / G_{5}\right| \leq p^{2}$.
\end{abstract}

Introduction. Our starting point is the set of metabelian two-generator groups of exponent $p$ and nilpotence class $c$, where $c \leq p-1$ and $p$ is an odd prime.

A specific group must be introduced. Set $F=\langle x, y\rangle$. Assume that $F$ is metabelian, of exponent $p$, and has nilpotence class $p-1$. Let $F_{2}, \ldots, F_{p}$ be the lower central series for $F$. Set

$$
u(i, j)=[y, x, \underbrace{\ldots}_{i-1-j}, x, y, \underbrace{\ldots}_{j}, y]
$$

for $i=2, \ldots, p-1$ and $j=0, \ldots, i-2$. Note that

$$
F_{i}=\left\langle u(i, 0), u(i, 1), \ldots, u(i, i-2), F_{i+1}\right\rangle
$$

for $i=2, \ldots, p-1$. Furthermore if we assume that the commutator subgroup $F_{2}$ is free then the set of all $u(i, j)$ is a basis for $F_{2},\{u(i, 0), u(i, 1), \ldots, u(i, i-2)\}$ is a basis for $F_{i}$ modulo $F_{i+1}$, and $\left|F_{i} / F_{i+2}\right|=p^{\gamma-1}$ for $i=2, \ldots, p-1$.

The group $F$ described in the preceding paragraph will be called the freemetabelian group of exponent $p$ and class $p-1$. The word "free" refers to the fact that the commutator subgroup $F_{2}$ is free. The group $F$ will serve as a frame for our discussion for if $G$ is any metabelian two-generator group of exponent $p$ and class at most $p-1$ then $G=F / M$ where $M$ is a normal subgroup of $F$ that is contained in $F_{2}$.

In the above, and in what follows, we assume the reader is familiar with the elementary theory. Chapters 1 and 3 of Huppert $[\mathbf{1}]$ are the basic references for our work.

The isomorphism classes for our groups are given by

THEOREM 1. Let $F$ be the free two-generator metabelian group of exponent $p$ and nilpotence class $p-1$ where $p$ is prime and $p \geq 3$. Let $F_{2}$ be the commutator subgroup of $F$ and $\Omega$ be the set of normal subgroups of $F$ contained in $F_{2}$. Let $\mathrm{GL}(2, p)$ be the set of nonsingular $2 \times 2$ matrices whose entries are elements of the field $p$ elements. Let $\mathfrak{G}$ be the set of isomorphism classes of the two-generator metabelian groups of exponent $p$ and class $c \leq p-1$. Then $\mathrm{GL}(2, p)$ is a permutation

Received by the editors May 3, 1984 .

1980 Mathematics Subject Classification. Primary 20D15; Secondary $20 \mathrm{~F} 12$.

Key words and phrases. p-groups, metabelian groups. 
group on $\Omega$ and the set of orbits of $\operatorname{GL}(2, p)$ on $\Omega$ is in one-to-one correspondence with the elements of $\mathfrak{G}$.

If $F=\langle x, y\rangle$ and $\theta=\left(\begin{array}{ll}\alpha & \beta \\ \gamma & \delta\end{array}\right)$ is in $\mathrm{GL}(2, p)$ then the action of $\theta$ on $\Omega$ is given as follows. Define an automorphism $\varsigma$ of $F$ by $x^{\varsigma}=x^{\alpha} y^{\beta}$ and $y^{\varsigma}=x^{\gamma} y^{\delta}$, so

$$
u(i, j)^{\varsigma}=[y^{\varsigma}, x^{\varsigma}, \underbrace{\ldots}_{i-1-j}, x^{\varsigma}, y^{\varsigma}, \underbrace{\ldots}_{j}, y^{\varsigma}] .
$$

Then if $N \in \Omega$ and $\theta$ is in $\operatorname{GL}(2, p)$, the action of $\theta$ is given by $N^{\theta}=\left\{g^{\varsigma}: g \in N\right\}$.

See $[\mathbf{3}]$ for the proof of Theorem 1 .

Theorem 1 is, at the present time, too general to give specific information. The quantities that arise there are, in principle, easy to compute. However, in practice, they are quite intractable. Thus we shall consider a special case in this paper.

To describe that case, let $G_{2}, \ldots, G_{c}$ be the lower central series for the group $G$. We shall study those groups $G$ where

$$
\left|G_{2} / G_{3}\right|=p, \quad\left|G_{3} / G_{4}\right|=p^{2}, \quad\left|G_{4} / G_{5}\right|=p^{2} .
$$

These assumptions are drawn to eliminate known cases and lead to nontrivial ones. For let $G=\langle y, x\rangle$ and $u=[y, x]$. We then have

$$
G_{2}=\left\langle u, G_{3}\right\rangle \text {. }
$$

Since $G$ is of exponent $p$ we must have $\left|G_{2} / G_{3}\right|=p$. Next, since $G$ is metabelian

$$
G_{3}=\left\langle[u, x],[u, y], G_{4}\right\rangle .
$$

Thus $\left|G_{3} / G_{4}\right| \leq p^{2}$. If $\left|G_{3} / G_{4}\right|=p$ then $G$ is of maximal class. These groups were classified in $[\mathbf{2}]$. Consequently, we assume that $\left|G_{3} / G_{4}\right|=p^{2}$. Finally

$$
G_{4}=\left\langle[u, x, x],[u, x, y],[u, y, y], G_{5}\right\rangle,
$$

so $\left|G_{4} / G_{5}\right| \leq p^{3}$. Thus the assumption that $\left|G_{4} / G_{5}\right|=p^{2}$ gives us one nontrivial commutator relation at this stage.

The following result is the first step in the classification of our groups.

THEOREM 2. Let $G$ be a two-generator metabelian group of exponent $p$ and class $c$ where $c \leq p-1$. Suppose that $\left|G_{4} / G_{5}\right|=p^{2}$. Then there is, up to multiplication by scalars, one nontrivial relation of the form

$$
u(4,0)^{a} u(4,1)^{b} u(4,2)^{c} \equiv 1 \quad \bmod G_{5} .
$$

In addition, we may assume this relation has one of three forms:

(a) $u(4,2) \equiv 1 \bmod G_{5}$,

(b) $u(4,1) \equiv 1 \bmod G_{5}$,

(c) $u(4,0)^{-d} u(4,2) \equiv 1 \bmod G_{5}$ where $d$ is a quadratic nonresidue modulo $p$.

Finally, relations (a), (b), and (c) split the groups $G$ into three disjoint classes with no two groups from two distinct classes being isomorphic.

Theorem 2 is one of the two main guidelines for our classification. The second consists of the fact that if $G$ satisfies the hypothesis of Theorem 2 then the factor groups $G_{i} / G_{i+1}$ satisfy one of two conditions: either

(i) $\left|G_{i} / G_{i+1}\right|=p^{2}, i=4, \ldots, c$, 
or there is an integer $r \leq c$ such that

(ii) $\left|G_{i} / G_{i+1}\right|=p^{2}, i=4, \ldots, r-1$, and $\left|G_{i} / G_{i+1}\right|=p, i=r, \ldots, c$.

We shall make a detailed study of the following cases. First, we assume condition (a) or (b) of Theorem 2 and condition (i) above hold. The detailed results for this case are given in $\S 3$. Second, we assume that the groups satisfy (ii) in the paragraph above and that $r<c$. The specific results for this case are given in $\S 4$.

A review of those specific results shows two persistent ideas. First, "most of the time" the groups under consideration have a pair of characteristic subgroups of index $p$ in the full group. Second, if the groups do not have a pair of characteristic subgroups of index $p$ then they are subject to a number of side conditions which, in turn, lead to the desired solution.

1. We shall prove Theorem 2 in this section.

In what follows $K$ is the field of $p$ elements, $p \geq 3$.

DEFINITION. Let $(a, b, c)$ be a triplet with $a, b, c \in K$. Let $\sim$ be the equivalence relation defined on the set of nonzero triplets by $(a, b, c) \sim(\bar{a}, \bar{b}, \bar{c})$ if and only if there is a nonsingular matrix $\left(\begin{array}{ll}\alpha & \beta \\ \gamma & \delta\end{array}\right)$ with entries in $K$ and a nonzero constant $\lambda \in K$ such that

$$
\left(\begin{array}{c}
\bar{a} \\
\bar{b} \\
\bar{c}
\end{array}\right)=\lambda\left(\begin{array}{ccc}
\alpha^{2} & \alpha \gamma & \gamma^{2} \\
2 \alpha \beta & \alpha \delta+\beta \gamma & 2 \gamma \delta \\
\beta^{2} & \beta \delta & \delta^{2}
\end{array}\right)\left(\begin{array}{l}
a \\
b \\
c
\end{array}\right) .
$$

The fact that $\sim$ is an equivalence relation follows from the fact that the map

$$
\left(\begin{array}{ll}
\alpha & \beta \\
\gamma & \delta
\end{array}\right) \rightarrow\left(\begin{array}{ccc}
\alpha^{2} & \alpha \gamma & \gamma^{2} \\
2 \alpha \beta & \alpha \delta+\beta \gamma & 2 \gamma \delta \\
\beta^{2} & \beta \delta & \delta^{2}
\end{array}\right)
$$

is an antiautomorphism.

DEFINITION. Let

$$
F_{2}[x, y]=\left\{a x^{2}+b x y+c y^{2}:(a, b, c) \neq(0,0,0)\right\} .
$$

Define an equivalence relation $\sim$ on $F_{2}[x, y]$ by $f(x, y) \sim g(x, y)$ if and only if there is a nonsingular matrix $\left(\begin{array}{c}\alpha \beta \\ \gamma \delta\end{array}\right)$ and a nonzero constant $\lambda$ such that

$$
g(x, y)=\lambda f(\alpha x+\beta y, \gamma x+\delta y) .
$$

LEMMA 1.1. Set $g(x, y)=\bar{a} x^{2}+\bar{b} x y+\bar{c} y^{2}$ and $f(x, y)=a x^{2}+b x y+c y^{2}$. Then (1) holds if and only if

$$
\left(\begin{array}{c}
\bar{a} \\
\bar{b} \\
\bar{c}
\end{array}\right)=\lambda\left(\begin{array}{ccc}
\alpha^{2} & \alpha \gamma & \gamma^{2} \\
2 \alpha \beta & \alpha \delta+\beta \gamma & 2 \gamma \delta \\
\beta^{2} & \beta \delta & \delta^{2}
\end{array}\right)\left(\begin{array}{l}
a \\
b \\
c
\end{array}\right) .
$$

PROOF. This can be verified by a straightforward computation.

LEMMA 1.2. Let $f(x, y)=a x^{2}+b x y+c y^{2}$. If $b^{2}-4 a c \equiv 0 \bmod p$ set $D(f)=0$. If $b^{2}-4 a c \not \equiv 0$ let $D(f)=1$ when $b^{2}-4 a c$ is a quadratic residue and $D(f)=-1$ when it is not. Then the equivalence relation defined above on $F_{2}[x, y]$ splits $F_{2}[x, y]$ into three equivalence classes $E_{i}$ where

$$
E_{i}=\{f(x, y): D(f)=i\}, \quad i=-1,0,1 .
$$


Proof. We first consider the case where $D(f) \neq 0$.

We have $f(x, y)=a x^{2}+b x y+c y^{2}$ with, since $D(f) \neq 0, a \neq 0$ or $c \neq 0$. We may assume $a \neq 0$. If not, interchange $x$ and $y$. Next we may assume $a=1$. The operations leading to the normalization " $a=1$ " do not affect the value of $D(f)$.

To continue, since $D(f) \neq 0$ the polynomial $f(x, 1)=x^{2}+b x+c$ has two distinct roots $r_{1}, r_{2}$ in $Z_{p}(\sqrt{d})$ where $d$ is any quadratic nonresidue. Thus we have

$$
f(x, y)=\left(x-r_{1} y\right)\left(x-r_{2} y\right) \quad r_{1} \neq r_{2} .
$$

The argument now splits into two cases. Suppose first that $r_{1}, r_{2} \in Z_{p}$. That is, $D(f)=+1$. We may suppose that $r_{2} \neq 0$. Set $\beta=r_{2} \delta$ and $\delta=-1 /\left(r_{2}-r_{1}\right)$. Then

$$
f(x+\beta y, \delta y)=(x-y) x .
$$

Consequently all polynomials with $D(f)=+1$ are equivalent to $(x-y) x$.

Suppose next that $D(f)=-1$. Then

$$
r_{1}=u+v \sqrt{d}, \quad r_{2}=u-v \sqrt{d}, \quad v \neq 0
$$

where $u$ and $v$ are in $Z_{p}$ and $d$ is a quadratic nonresidue. Set $\beta=\delta u$ and $\delta=Y v$. Then

$$
f(x+\beta y, \delta y)=x^{2}-d y^{2} .
$$

Thus all the polynomials with $D(f)=-1$ are equivalent to $x^{2}-d y^{2}$.

The case where $D(f)=0$ remains. We have

$$
f(x, y)=a x^{2}+b x y+c y^{2}
$$

with $(a, b, c) \neq(0,0,0)$ and $b^{2}-4 a c=0$. Under these assumptions we must have $a \neq 0$ or $c \neq 0$, so we may assume that $a=1$. Thus $f(x, y)=x^{2}+b x y+c y^{2}$ with $b^{2}-4 c=0$. Since $c=b^{2} / 4$ we have

But then

$$
f(x, y)=\left(x+\frac{b}{2} y\right)^{2} .
$$

$$
f\left(x-\frac{b}{2} y, y\right)=x^{2},
$$

which completes the proof of Lemma 2.2 .

PROOF OF THEOREM 2. Since

$$
G_{4}=\left\langle[y, x, x, x],[y, x, x, y],[y, x, y, y], G_{5}\right\rangle
$$

and $\left|G_{4} / G_{5}\right|=p^{2}$ there is-up to multiplication by scalars-exactly one nontrivial relation among the commutators modulo $G_{5}$, say

$$
[y, x, x, x]^{a}[y, x, x, y]^{b}[y, x, y, y]^{c} \equiv 1 \bmod G_{5} .
$$

We work modulo $G_{5}$ in what follows.

Let $\{\bar{x}, \bar{y}\}$ be another pair of generators of $G$. Then there is a nonzero triplet $(\bar{a}, \bar{b}, \bar{c})$ such that

$$
[\bar{y}, \bar{x}, \bar{x}, \bar{x}]^{\bar{a}}[\bar{y}, \bar{x}, \bar{x}, \bar{y}]^{\bar{b}}[\bar{y}, \bar{x}, \bar{y}, \bar{y}]^{\bar{c}} \equiv 1 .
$$

In addition

$$
\bar{x}=x^{\alpha} y^{\beta}, \quad \bar{y}=x^{\gamma} y^{\delta}
$$

where $\alpha \delta-\beta \gamma=\Delta \neq 0$. 
Now $[\bar{y}, \bar{x}]=[y, x]^{\Delta} g$ where $g \in G_{3}$. Thus, from (3),

$$
\left\{[y, x], x^{\alpha} y^{\beta}, x^{\alpha} y^{\beta}\right]^{\bar{a}}\left\{[y, x], x^{\alpha} y^{\beta}, x^{\gamma} y^{\delta}\right]^{\bar{b}}\left\{[y, x], x^{\gamma} y^{\delta}, x^{\gamma} y^{\delta}\right]^{\bar{c}} \equiv 1 .
$$

If we expand this by the usual commutator rules and compare the result with (2) we obtain

$$
\left(\begin{array}{ccc}
\alpha^{2} & \alpha \gamma & \gamma^{2} \\
2 \alpha \beta & \alpha \delta+\beta \gamma & 2 \gamma \delta \\
\beta^{2} & \beta \delta & \delta^{2}
\end{array}\right)\left(\begin{array}{l}
\bar{a} \\
\bar{b} \\
\bar{c}
\end{array}\right)=\lambda\left(\begin{array}{l}
a \\
b \\
c
\end{array}\right) .
$$

Consequently the three-tuples $(a, b, c)$ split into three equivalence classes. We can choose $(0,0,1),(0,1,1)$ and $(-d, 0,1)$ where $d$ is a quadratic nonresidue, as representatives of these classes. This gives us the defining relations of Theorem 2 .

2. We shall introduce a convenient basis for the commutator subgroup $F_{2}$ of $F$ in this section. In addition we shall list several known results that will be employed in the sequel.

Let $S_{n}^{m}$ be a Stirling number of the first kind. It is defined by

$$
(x)_{n}=x(x-1) \cdots(x-n+1)=\sum_{m=0}^{n} S_{n}^{m} x^{m} .
$$

Let $\sigma(m, n)=(m ! / n !) S_{n}^{m}$ and

$$
s(i, j, q, r)=\sigma(j+1, r+1) \sigma(i-j-1, q-r-1) .
$$

Define $U(i, j)$ by

$$
U(i, j)=\prod_{q=i}^{p-1} \prod_{r=j}^{j+q-i} u(q, r)^{s(i, j, q, r)} .
$$

Note that since $s(i, j, i, j)=1$ we have $U(i, j) \equiv u(i, j) \bmod G_{i+1}$. Consequently the set of all $U(i, j)$ form a basis for $F_{2}$ and $\{U(i, 0), \ldots, U(i, i-2)\}$ is a basis for $F_{i}$ modulo $F_{i+1}$. We shall also see that if $v$ is the map from $F$ to $F$ defined by $x^{v}=x^{\alpha}$ and $y^{v}=y^{\delta}$ then $U(i, j)=U(i, j)^{s(i, j)}$ where $s(i, j)=\alpha^{i-j-1} \delta^{j-2}$.

More generally, we have

LEMMA 2.1. Let $F$ be the two-generator, free-metabelian group of exponent $p$ and class $p-1$. Let $v$ be the automorphism of $F$ defined by

$$
x^{v}=x^{\alpha} y^{\beta}, \quad y^{v}=y^{\delta} .
$$

Let $U(i, j)$ be defined as above. Then for $i=2, \ldots, p-1$ and $j=0, \ldots, i-2$,

$$
U(i, j)^{v}=\prod_{q=i}^{p-1} \prod_{r=j}^{q-2} U(q, r)^{f(i, j, q, r)}
$$

where

$$
\begin{gathered}
f(i, j, q, r)=\alpha^{q-r-1} \beta^{r-j} \delta^{j+1} g(i, j, q, r), \\
g(i, j, q, r)=\left(\begin{array}{c}
q-j-1 \\
q-r-1
\end{array}\right) \delta(q, i)-\sum_{m=0}^{q-i} \frac{B_{m}}{(q-i-1-m) !}\left(\begin{array}{c}
m+i-j-2 \\
q-r-1
\end{array}\right),
\end{gathered}
$$

$\delta(q, i)$ is Kronecker's delta and $B_{m}$ is the mth Bernoulli number. 
See [4] for a proof.

Incidentally, the Bernoulli numbers $B_{m}$ are defined by $B_{0}=1$ and

$$
\sum_{j=0}^{n} \frac{B_{j}}{(n+1-j) !}=0
$$

for $n \geq 1$.

There are two particular values of the functions $f(i, j, q, r)$ that we shall need.

LEMMA 2.2. We have

$$
f(i, j, q, j)=\delta(q, i) \alpha^{q-j-1} \delta^{j+1}
$$

and

$$
f(i, j, q, j+1)=\left[(q-j-1) \delta(q, i)-B_{q-i}\right] \alpha^{q-j-2} \delta^{j+2} \beta
$$

where $\delta(q, i)=1$ if $i=q$ and $\delta(q, i)=0$ if $q \neq i$.

This follows directly from the definitions.

LEMMA 2.3. Suppose $U(i, j)$ is defined as in the introduction and

$$
U(q, r)=\prod_{i} \prod_{j} U(i, j)^{B(i, j)}
$$

where $q, r$, the $(i, j)$ and the $B(i, j)$ are arbitrary fixed integers. Let $M$ be any fixed nonnegative integer. Then

$$
U(q+M, r)=\prod_{i} \prod_{j} U(i+M, j)^{B(i, j)}
$$

and

$$
U(q+M, r+M)=\prod_{i} \prod_{j} U(i+M, j+M)^{B(i, j)} .
$$

We do not specify the range of the indices, $i$ and $j$, in Lemma 1.1 because the result holds for any finite range one might select.

3. In this section we shall study those groups where $\left|G_{2} / G_{3}\right|=p$ and

$$
\left|G_{i} / G_{i+1}\right|=p^{2}, \quad i=3, \ldots, c .
$$

We shall consider the subcases of Theorem 2 where $u(4,2) \equiv 1 \bmod G_{5}$ and $u(4,1) \equiv$ $1 \bmod G_{5}$.

Our first result treats the case where $u(4,2) \equiv 1 \bmod G_{5}$. Passing from the $u(i, j)$ basis to the $U(i, j)$ basis of $F_{2}$ we have

THEOREM 3.1. Suppose that $G=\langle x, y\rangle$ satisfies the hypothesis of Theorem 2, $\left|G_{i} / G_{i+1}\right|=p^{2}$ for $i=4, \ldots, c$, and $u(4,2) \equiv 1 \bmod G_{5}$. Set $H=\left\langle y, G_{2}\right\rangle$. Then $H$ is a characteristic subgroup of index $p$ in $G$. Let $U(i, j)$ be defined as above. Then

$$
G_{i}=\left\langle U(i, 0), U(i, 1), G_{i+1}\right\rangle
$$

for $i=4, \ldots, c$. Thus

$$
U(4,2)=\prod_{i=k}^{c} \prod_{j=0}^{1} U(i, j)^{A(i, j)}
$$


where $k$ is some integer $\geq 5$ defined by the condition $[A(k), B(k)] \neq(0,0)$. Next:

(a) Suppose there is an integer $q$ with $k \leq q \leq 2 k-5$ such that $A(\nu, 0)=0$ for $\nu \leq q-1$ and $A(q, 0) \neq 0$. Then there is an $x$ in $G$ such that $G=\langle x, y\rangle$, where $H=\left\langle y, G_{2}\right\rangle$, and such that $A(q, 1)=0$.

(b) Suppose that $A(\nu, 0)=0$ for $\nu=k, \ldots, 2 k-5$. Set

$$
B(\nu)=(\nu-2) A(\nu, 0)+\sum_{m=k}^{\nu+4-k}(m-3) A(m, 1) A(\nu+4-m, 1)
$$

for $\nu \geq 2 k-4$. Suppose there is an integer $q$ with $2 k-4 \leq q \leq 3 k-q$ such that $B(\nu)=0$ for $\nu \leq q-1$ and $B(q) \neq 0$. Then there is an $x$ in $G$ such that $G=\langle x, y\rangle$ and such that $A(q, 1)=0$.

(c) Suppose that $B(\nu)=0$ for $\nu=2 k-4, \ldots, 3 k-q$. Suppose also that $3 k-8 \leq c$. Then there is an $x$ in $G$ such that $G=\langle x, y\rangle$ and $A(3 k-8,0)=0$.

To continue, if in (a) and (b) (or (c)) $X$ is the set of elements $x$ such that $G=\langle x, y\rangle$, where $H=\left\langle y, G_{2}\right\rangle$, and such that $A(q, 1)=0($ or $A(3 k-8,0)=0)$ then $\left\langle x, G_{2}\right\rangle$ is a characteristic subgroup of index $p$ in $G$. Finally if $\bar{G}$ is a group satisfying the assumptions of Theorem 3.1 and is defined in terms of the parameters $\bar{A}(i, j)$ then $\bar{G}$ is isomorphic to $G$ if and only if there are integers $\alpha$ and $\delta$ with $(\alpha \delta, p)=1$ such that

$$
\delta^{2-j} A(i, j)=\alpha^{i-j-2} \bar{A}(i, j)
$$

for $j=0, \ldots, 1$ and $i=k, \ldots, c$.

In Theorem 3.1 we assume that $U(4,2) \neq 1$; thus $k \leq c$. There is also one group where $U(4,2)=1$.

As for the case where $U(4,1) \equiv 1 \bmod G_{5}$, we have

THEOREM 3.2. Suppose that $G=\langle x, y\rangle$ satisfies the hypothesis of Theorem 2, $\left|G_{i} / G_{i+1}\right|=p^{2}$ for $i=4, \ldots, c$ and $u(4,1) \equiv 1 \bmod G_{5}$. Then

$$
G_{i}=\left\langle U(i, 0), U(i, i-2), G_{i+1}\right\rangle
$$

for $i=4, \ldots, c$. Consequently

$$
U(4,1)=\prod_{i \geq 5} U(i, 0)^{A(i)} U(i, i-2)^{B(i)} .
$$

Next, let $\bar{G}$ be a group that satisfies the hypothesis of this theorem. Then $G$ is isomorphic to $\bar{G}$ if and only if one of the following two conditions hold.

(a) There is a pair of integers $\alpha, \delta$ with $(\alpha \delta, p)=1$ such that $\delta A(i)=\alpha^{i-3} \bar{A}(i)$, $\alpha B(i)=\delta^{i-3} \bar{B}(i), i=4, \ldots, c$.

(b) There is a pair of integers $\gamma, \beta$ with $(\gamma \beta, p)=1$ such that $\beta A(i)=\gamma^{i-3} \bar{B}(i)$, $\gamma \beta(i)=\beta^{i-3} \bar{A}(i), i=5, \ldots, c$.

The statement that the subgroup $H$ of Theorem 3.1 is a characteristic subgroup is easy to prove. We have

$$
u(4,2)=\langle[y, x], y, y\rangle \equiv 1 \bmod G_{5} .
$$

Let $x^{v}=x^{\alpha} y^{\beta}, y^{v}=x^{\gamma} y^{\delta}$ be an automorphism of $G$. Setting these values in the relation above we find that we must have $\gamma=0$. Thus $H=\left\langle y, G_{2}\right\rangle$ is a characteristic subgroup of $G$. 
Next, since $u(4,2) \equiv 1 \bmod G_{5}$ we have $u(i, j) \equiv 1 \bmod G_{i+1}$ for $i \geq 4$ and $j=2, \ldots, i-2$. Thus $G_{i}=\left\langle u(i, 0), u(i, 1), G_{i+1}\right\rangle$. Since $U(i, j) \equiv u(i, j) \bmod G_{i+1}$ it follows that $G_{i}=\left\langle U(i, 0), U(i, 1), G_{i+1}\right\rangle$.

LEMMA 3.1. Let $A(i, j)$ be as in Theorem 3.1. Set $A(i, j)=0$ when $(i, j)$ is not in the range $i=k, \ldots, c ; j=0,1$. Let

$$
E_{1}(m, q, \nu, j)=\sum_{a=2}^{q+1} \sum_{r=0}^{q+1-a} \sum_{i} \sum_{x} A\left(x_{1}, j-r\right) A\left(x_{2}, 2-i_{2}\right) \cdots A\left(x_{a}, 2-i_{2}\right)
$$

where the summation is on those $i=\left(i_{2}, \ldots, i_{a}\right)$ and $x=\left(x_{1}, \ldots, x_{a}\right)$ where $i_{2}+$ $\cdots+i_{a}=q-r$ and $x_{1}+\cdots+x_{a}=\nu-m+4 a$. Let

$$
E(m, q, \nu, j)=A(\nu-m+4, j-q)+E_{1}(m, q, \nu, j) .
$$

Then for $q \geq 0$ and $m \geq q+4$

$$
U(m, 2+q)=\prod_{j=0}^{1} \prod_{\nu \geq m+k-4} U(\nu, j)^{E(m, q, \nu, j)} .
$$

PROOF. This follows by induction from the value of $U(4,2)$ given in Theorem 3.1. Further details on the proof can be found in $\S 1$ of $[4]$.

In what follows $G=\langle x, y\rangle$ and $\bar{G}=\langle\bar{x}, \bar{y}\rangle$ are groups that satisfy the assumptions of Theorem 3.1. We suppose that

$$
\bar{U}(4,2)=\prod_{i \geq k} \prod_{j=0}^{1} \bar{U}(i, j)^{\bar{A}(i, j)} .
$$

We also suppose that $v$ is the map from $\bar{G}$ to $G$ defined by $\bar{x}^{v}=x^{\alpha} y^{\beta}, \bar{y}^{v}=y^{\delta}$.

LEMMA 3.2. Set

$$
L(\nu, j)=\sum_{m=4}^{\nu-k+4} \sum_{q=0}^{m-4} f(4,2, m, q+2) E(m, q, \nu, j)
$$

Then

$$
\bar{U}(4,2)^{v}=\prod_{j=0}^{1} \prod_{\nu \geq k} U(\nu, j)^{L(\nu, j)}
$$

PROOF. To start, apply Lemma 2.1 to get

$$
\bar{U}(4,2)^{v}=\prod_{m \geq 4} \prod_{q=0}^{m-4} U(m, 2+q)^{f(4,2, m, 2+q)} .
$$

To finish, apply Lemma 3.1.

LEMMA 3.3. Set

$$
R_{0}(\nu)=\sum_{i=k}^{\nu} f(i, 0, \nu, 0) \bar{A}(i, 0)
$$

and

$$
R_{1}(\nu)=\sum_{i=k}^{\nu}[f(i, 1, \nu, 1) \bar{A}(i, 1)+f(1,0, \nu, 1) \bar{A}(i, 0)]
$$


Let, for $j=0,1$,

$$
S_{j}(\nu)=\sum_{m=k}^{\nu-k+4} \sum_{q=0}^{m-4} \sum_{i=k}^{m} \sum_{t=0}^{1} f(i, t, m, q+2) \bar{A}(i, t) E(m, q, \nu, j) .
$$

Then

$$
\prod_{j=0}^{1} \prod_{i=k}^{c}\left(\bar{U}(i, j)^{v}\right)^{\bar{A}(i, j)}=\prod_{j=0}^{1} \prod_{\nu \geq k} U(\nu, j)^{\left(R_{j}(\nu)+s_{j}(\nu)\right)} .
$$

PROOF. By Lemma 2.1, the left side above is equal to

$$
\prod_{i \geq k} \prod_{j=0}^{1} \prod_{m \geq k} \prod_{r=j}^{m-2} U(m, r)^{f(i, j, m, r) \bar{A}(i, j)} .
$$

This can be written as

$$
\prod_{\nu \geq k} U(\nu, 0)^{R_{0}(\nu)} \prod_{\nu \geq k} U(\nu, 1)^{R_{1}(\nu)} \prod_{q \geq 0} \prod_{m \geq k} U(m, 2+q)^{T(m, 2+q)}
$$

where

$$
T(m, 2+q)=\sum_{i=k}^{m} \sum_{t=0}^{1} f(i, t, m, 2+q) \bar{A}(i, t) .
$$

To complete the proof apply Lemma 3.1.

Lemma $3.3^{*}$. Suppose that $G$ is isomorphic to $\bar{G}$. Then for $\nu=k, \ldots, 2 k-5$,

$$
\alpha \delta^{3} A(\nu, 0)=\alpha^{\nu-1} \delta \bar{A}(\nu, 0)
$$

and

$$
\begin{aligned}
\alpha \delta^{3} A(\nu, 1)+\sum_{i=k}^{\nu} f(4,2, \nu+4-i, 3) A(\nu, 0) \\
=\alpha^{\nu-2} \delta^{2} \bar{A}(\nu, 1)+\sum_{i=k}^{\nu} f(i, 0, \nu, 1) \bar{A}(i, 0) .
\end{aligned}
$$

ProOF. This follows from Lemmas 3.2 and $3.3^{*}$.

As for the details, when $\nu \leq 2 k-5$ we have $E(m, q, \nu, j)=A(\nu-m+4, j-q)$ so

$$
L(\nu, j)=\sum_{m=4}^{\nu-k+4} \sum_{q=0}^{m-4} f(4,2, m, q+2) A(\nu-m+4, j-q) .
$$

When $j=0$ we obtain

$$
L(\nu, 0)=\sum_{m=4}^{\nu-k+4} f(4,2, m, 2) A(\nu-m+4,0)=f(4,2,4,2) A(\nu, 0) .
$$

Then, by Lemma $2.2, f(4,2,4,2)=\alpha \delta^{3}$. When $j=1$,

$$
L(\nu, 1)=f(4,2,4,2) A(\nu, 1)+\sum_{i=k}^{\nu} f(4,2, \nu+4-i, 3) A(\nu, 0) .
$$

This gives us the left side of our equations. The right side comes from Lemma 3.3. 
LEMMA 3.4. Suppose there is an integer $q$ with $k \leq q \leq 2 k-5$ such that $A(\nu, 0)=0$ for $\nu \leq q-1$ and $A(q, 0) \neq 0$. Then $\alpha \delta^{3} A(q, 0)=\alpha^{q-1} \delta \bar{A}(q, 0)$ and

$$
\alpha \delta^{3} A(q, 1)=\alpha^{q-1} \delta^{2} \bar{A}(q, 1)+(q-2) \alpha^{q-2} \delta \bar{A}(q, 0) \beta .
$$

PROOF. This is a direct consequence of Lemma $3.3^{*}$.

Part (a) of Theorem 3.1 follows from Lemma 3.4. First we normalize by taking $A(q, 1)=0=\bar{A}(q, 1)$. Then we must have $\beta=0$. Thus we obtain the specified characteristic subgroup.

Lemma 3.5. Suppose that $A(i, 0)=0$ for $i=k, \ldots, 2 k-5$. Then for $\nu \leq 3 k-9$,

$$
E_{1}(m, 1, \nu, j)=\sum_{x_{1}+x_{2}=\nu-m+8} A\left(x_{1}, j\right) A\left(x_{2}, 1\right)
$$

and $E_{1}(m, q, \nu, j)=0$ for $q \geq 2$.

PROOF. By our definitions

$$
E_{1}(m, q, \nu, j)=\sum_{r=0}^{q-1} \sum_{x_{1}+x_{2}=\nu-m+8} A\left(x_{1}, j-r\right) A\left(x_{2}, 1\right)
$$

when $\nu \leq 3 k-9$. Furthermore if the sum is to be nontrivial we must have $j-r=1$ and $q-r=1$. Since $j=0$ or 1 this occurs only when $q=1$ and $j=1$.

LEMMA 3.6. Suppose that $\nu \leq 3 k-9$. Then

$$
\begin{aligned}
L(\nu, 1)= & \alpha \delta^{3} A(\nu, 1) \\
& +\sum_{m=4}^{\nu-2 k+8} f(4,2, m, 3)\left[A(\nu-m+4,0)+\sum_{x_{1}+x_{2}=\nu-m+8} A\left(x_{1}, 1\right) A\left(x_{2}, 1\right)\right]
\end{aligned}
$$

and

$$
L(\nu, 0)=\alpha \delta^{3} A(\nu, 0) .
$$

PROOF. This follows from the definitions and Lemma 3.5.

LemMa 3.7. Suppose that $\nu \leq 3 k-9$. Let $S_{j}(\nu)$ be defined as in Lemma 3.3. Then $S_{0}(\nu)=0$ and

$$
S_{1}(\nu)=\sum_{m=k}^{\nu-k+4} \sum_{i=k}^{m} f(i, 1, m, 2) A(\nu-m+4,1) \bar{A}(i, 1) .
$$

PROOF. By definition

$$
S_{j}(\nu)=\sum_{m=k}^{\nu-k+4} \sum_{q=0}^{m-4} \sum_{i=k}^{m} \sum_{t=0} f(i, t, m, q+2) \bar{A}(i, t) E(m, q, \nu, j) .
$$

Now if $t=0$ here we have $\nu-k+4 \geq m \geq i \geq k \geq 2 k-4$, so $\nu \geq 2 k-8$. Consequently

$$
S_{j}(\nu)=\sum_{m=k}^{\nu-k+4} \sum_{q=0}^{m-4} \sum_{i=k}^{m} f(i, 1, m, q+2) \bar{A}(i, t)\left[A(\nu-m+4, j-q)+E_{1}(m, q, \nu, j)\right] .
$$


Now, referring to that part of the sum corresponding to $A(\nu-m+4, j-q)$ : if $q \geq 1$ then $\nu-m+4 \geq 2 k-4$, so $\nu \geq 3 k-8$. Thus

$$
\begin{aligned}
S_{j}(\nu)= & \sum_{m=k}^{\nu-k+4} \sum_{i=k}^{m} f(i, 1, m, 2) \bar{A}(i, t) A(\nu-m+4, j) \\
& +\sum_{m=k}^{\nu-k+4} \sum_{i=k}^{m} f(i, 1, m, q+2) \bar{A}(i, t) \sum_{x_{1}+x_{2}=\nu-m+8} A\left(x_{1}, j\right) A\left(x_{2}, 1\right) .
\end{aligned}
$$

Finally, in the last sum, we have $\nu-m+8=x_{1}+x_{2} \geq 2 k$ so it too is trivial.

LEMMA 3.8. Suppose that $G$ is isomorphic to $\bar{G}$ and $A(i, 0)=0$ for $i \leq 2 k-5$. Then for $\nu=k, \ldots, 3 k-9$

$$
\alpha \delta^{3} A(\nu, 0)=\alpha^{\nu-1} \delta \bar{A}(\nu, 0)
$$

and

$$
\begin{gathered}
\alpha \delta^{3} A(\nu, 1)+\sum_{i=2 k-4}^{\nu} f(4,2, \nu+4-i, 3)\left[A(i, 0)+\sum_{x_{1}+x_{2}=i+4} A\left(x_{1}, 1\right) A\left(x_{2}, 1\right)\right] \\
=\alpha^{\nu-2} \delta^{2} \bar{A}(\nu, 1)+\sum_{i=2 k-4}^{\nu} f(i, 0, \nu, 1) \bar{A}(i, 0) \\
+\sum_{m=k}^{\nu-k+4} \sum_{i=k}^{m} f(i, 1, m, 2) A(\nu-m+4,1) \bar{A}(i, 1) .
\end{gathered}
$$

PROOF. This follows from preceding results.

LEMMA 3.9 .

$$
\begin{aligned}
& \sum_{i=2 k-4}^{\nu} f(4,2, \nu+4-i, 3)\left[A(i, 0)+\sum_{x_{1}+x_{2}=i+4} A\left(x_{1}, 1\right) A\left(x_{2}, 1\right)\right] \\
& =-\sum_{i=2 k-4}^{\nu-1} B_{\nu-i} \alpha^{\nu-i} \delta^{3} \beta\left[A(i, 0)+\sum_{x_{1}+x_{2}=i+4} A\left(x_{1}, 1\right) A\left(x_{2}, 1\right)\right] .
\end{aligned}
$$

ProOF. By Lemma 1.2,

$$
f(4,2, \nu+4-i, 3)=\left[(\nu+1-i) \delta(\nu+4-i, 4)-B_{\nu-i}\right] \alpha^{\nu-1} \delta^{3} \beta .
$$

LEMMA 3.10 .

$$
\sum_{i=2 k-4}^{\nu} f(i, 0, \nu, 1) \bar{A}(i, 0)=\sum_{i=2 k-4}\left[(\nu-1) \delta(\nu, i)-B_{\nu-1}\right] \alpha^{\nu-1} \delta^{3} \beta A(i, 0) .
$$

PROOF. First, by Lemma 1.2,

$$
f(i, 0, \nu, 1)=\left[(\nu-1) \delta(\nu, i)-B_{\nu-i}\right] \alpha^{\nu-2} \delta \beta .
$$

Then, by Lemma 3.8

$$
\delta \bar{A}(i, 0)=\alpha^{-i+2} \delta^{3} A(i, 0)
$$


LEMMA 3.11 .

$$
\begin{aligned}
\sum_{m=k}^{\nu-k+4} \sum_{i=k}^{m} f(i, 1, m, 2) A( & -m+4,1) \bar{A}(i, 1) \\
= & \delta^{3} \beta \sum_{x_{1}+x_{2}=\nu+4}\left(x_{2}-3\right) A\left(x_{1}, 1\right) A\left(x_{2}, 1\right) \\
& -\sum_{i=2 k-4}^{\nu-1} B_{\nu-i} \alpha^{\nu-i} \delta^{2} \beta \sum_{x_{1}+x_{2}=\nu+4} A\left(x_{1}, 1\right) A\left(x_{2}, 1\right) .
\end{aligned}
$$

PROOF. The initial sum can be rearranged as

$$
\sum_{i=2 k-4}^{\nu} \sum_{x_{1}+x_{2}=i+4} f\left(x_{2}, 1, x_{2}+\nu-i, 2\right) A\left(x_{1}, 1\right) \bar{A}\left(x_{2}, 1\right) .
$$

Then taking $i=\nu$ we get the first sum of the conclusions. The second sum comes from those $i \leq \nu-1$.

LEMMA 3.12. If $G$ is isomorphic to $\bar{G}$ we have, for $\nu=k, \ldots, 3 k-9$,

$$
\alpha \delta^{3} A(\nu, 0)=\alpha^{\nu-1} \delta \bar{A}(\nu, 0)
$$

and

$$
\begin{aligned}
\alpha \delta^{3} A(\nu, 1)= & \alpha^{\nu-2} \delta^{2} \bar{A}(\nu, 1) \\
& +\delta^{3} \beta(\nu-2) A(\nu, 0)+\sum_{x_{1}+x_{2}=\nu+4}\left(x_{2}-3\right) A\left(x_{1}, 1\right) A\left(x_{2}, 1\right) .
\end{aligned}
$$

Proof. This follows from the last four lemmas.

Part (b) of Theorem 3.1 now follows from Lemma 3.12.

LEMMA 3.13. We have

$$
E(m, 0,3 k-8,0)=A(3 k-4-m, 0) .
$$

For $q \geq 1$ and $m \geq 4, E(m, q, 3 k-8,0)=0$ except in the case $m=4$ and $q=1$. In this case $E(4,1,3 k-8,0)=A(2 k-4,0) A(k, 1)$.

PROOF. By our definitions

$$
E(m, q, 3 k-8,0)=A(3 k-4-m, 0-q)+E_{1}(m, q, 3 k-8,0)
$$

with

$$
E_{1}(m, q, 3 k-8,0)=\sum_{a=2}^{q+1} \sum_{i_{2}+\cdots+i_{a}=q} \sum_{x} A\left(x_{1}, 0\right) A\left(x_{2}, 2-i_{2}\right) \cdots A\left(x_{a}, 2-i_{a}\right)
$$

where $x=\left(x_{1}, \ldots, x_{a}\right)$ with $x_{1}+\cdots+x_{a}=3 k-8-m+4 a$.

Taking $q=0$ we get the first quantity of Lemma 3.13. Taking $q=1$ we obtain

$$
E_{1}(m, q, 3 k-8,0)=\sum_{x_{1}+x_{2}=3 k-m} A\left(x_{1}, 0\right) A\left(x_{2}, 2-q\right) .
$$

The only time this is not zero is when $q=1$ and $m=4$. 
LEMMA 3.14. We have

$$
L(3 k-8,0)=f(4,2,4,2) A(3 k-8,0)+f(4,2,4,3) A(2 k-4,0) A(k, 1) .
$$

PROOF. This follows from the definition of $L(v, j)$ given in Lemma 3.2 and Lemma 3.13.

LEMMA 3.15. We have

$$
R_{0}(3 k-8)=f(3 k-8,0,3 k-8,0) \bar{A}(3 k-8,0)
$$

and

$$
S_{0}(3 k-8)=f(k, 1, k, 2) A(2 k-4,0) \bar{A}(k, 1) .
$$

PROOF. This follows from Lemmas 3.3 and 3.13.

LEMMA 3.16. Suppose that $G$ is isomorphic to $\bar{G}$. Then for $\nu=3 k-8$ and $j=0$ we have

$$
\alpha \delta^{3} A(3 k-8,0)=\alpha^{3 k-9} \delta \bar{A}(3 k-8,0)+(k-3) \alpha^{k-3} \delta^{2} \beta A(2 k-4,0) \bar{A}(k, 1) .
$$

PROOF. This is a consequence of the last two lemmas.

Part (c) of Theorem 3 follows from Lemma 3.16. Since

$$
B(2 k-4)=(2 k-6) A(2 k-4,0)+(k-3) A(k, 1) A(k, 1)=0
$$

and since $A(k, 1) \neq 0$ we have $A(2 k-4,0) \neq 0$. Thus we can normalize the $A(3 k-8,0)$ parameter.

This completes the proof of Theorem 3.1, so we turn to the proof of Theorem 3.2 .

LEMMA 3.17. Suppose that $G=\langle x, y\rangle$ satisfies the hypothesis of Theorem 3.2. Let $\bar{G}\langle\bar{x}, \bar{y}\rangle$ be a group isomorphic to $G$ and

$$
\bar{x}^{v}=x^{\alpha} y^{\beta}, \quad \bar{y}^{v}=x^{\gamma} y^{\delta}
$$

be the connecting isomorphism. Then $\beta=\gamma=0$ or $\alpha=\delta=0$. That is, $\nu$ has one of two forms: $\bar{x}^{v}=x^{\alpha}, \bar{y}^{v}=y^{\delta}$ or $\bar{x}^{v}=y^{\beta}, \bar{y}^{v}=x^{\gamma}$.

Proof. By assumption, $\bar{u}(4,1) \equiv 1 \bmod G_{5}$. Thus if we let $\Delta=\alpha \delta-\beta \gamma$ we have

$$
\bar{u}(4,1) \equiv\left[u^{\Delta}, x^{\gamma} y^{\delta}, x^{\alpha} y^{\beta}\right] \equiv 1 \bmod G_{5} .
$$

Thus

$$
[u, x, x]^{\alpha \gamma}[u, x, y]^{(\alpha \delta+\beta \gamma)}[u, y, y]^{\delta \beta} \equiv 1 \bmod G_{5} .
$$

Since $\left|G_{4} / G_{5}\right|=p^{2}$ and $u(4,1)=[u, x, y] \equiv 1 \bmod G_{5}$ we must have $\alpha \gamma=0$ and $\delta \beta=0$. Thus $\beta=\gamma=0$ or $\alpha=\delta=0$.

LEMMA 3.18. Suppose that $G$ satisfies the hypothesis of Theorem 3.2. Then

$$
G_{i}=\left\langle U(i, 0), U(i, i-2), G_{i+1}\right\rangle
$$

for $i=4, \ldots, c$.

PRoOF. Since $u(4,1) \equiv 1 \bmod G_{5}$ and $\left|G_{i} / G_{i+1}\right|=p^{2}$ for $i \geq 4$ we have

$$
G_{i}=\left\langle u(i, 0), u(i, i-2), G_{i+1}\right\rangle .
$$

In addition, $U(i, j) \equiv u(i, j) \bmod G_{i+1}$. 
LEMma 3.19. Let $F=\langle x, y\rangle$ be the free-metabelian group of exponent $p$ and class $p-1$. Let $v$ be the automorphism of $F$ defined by $x^{v}=y^{\beta}, y^{v}=x^{\gamma}$. Then

$$
U(i, j)^{v}=U(i, i-2-j)^{-\tau(i, j)}
$$

where $\tau(i, j)=\gamma^{j+1} \beta^{i-j-1}$.

PROOF. By our definitions

$$
U(i, j)^{v}=\prod_{q \geq i}^{j+q-i} \prod_{r=j}^{j}[\left[y^{\beta}, x^{\alpha}\right], x^{\alpha}, \underbrace{\ldots}_{r}, x^{\alpha}, y^{\beta}, \underbrace{\ldots}_{q-2-r}, y^{\beta}]^{-\sigma(j+1, r+1) \sigma(i-j-1, q-r-1)} .
$$

Second, by the definition of the $U(i, j)$,

$$
U(i, i-2-j)=\prod_{q \geq i} \prod_{r=j}^{j+q-i} u(q, q-2-r) \sigma^{(j+1, r+1)} \sigma^{(i-j-1, q-r-1)} .
$$

Thus, if we define $\theta$ on $F$ by $x^{\theta}=x^{\gamma}, y^{\theta}=y^{\beta}$ then

$$
U(i, j)^{v}=\left(U(i, i-2-j)^{\theta}\right)^{-1} .
$$

Finally, for maps of the form $\theta$

$$
U(i, i-2-j)=U(i, i-2-j)^{\tau(i, j)} .
$$

Theorem 3.2 now follows from Lemmas 3.17 through 3.19.

4. In this section we shall consider those groups $G$ where $\left|G_{2} / G_{3}\right|=p$,

$$
\begin{array}{ll}
\left|G_{i} / G_{i_{1}}\right|=p^{2}, & i=3, \ldots, r-1, \\
\left|G_{i} / G_{i+1}\right|=p, & i=r, \ldots, c,
\end{array}
$$

and $r<c$. Our main results are stated as Theorems 4.1 through 4.4 .

THEOREM 4.1. Let $G$ be a metabelian, two-generator group of exponent $p$ and class $c$ where $c \leq p-1$. Suppose that $\left|G_{2} / G_{3}\right|=p,\left|G_{i} / G_{i+1}\right|=p^{2}$ for $i=$ $3, \ldots, r-1,\left|G_{i} / G_{i+2}\right|=p$ for $i=r, \ldots, c$ and $r<c$. Let $H$ be the largest subgroup of $G$ such that $\left[H, G_{r}\right] \leq G_{r+2}$. Then $H$ is a characteristic subgroup of index $p$ in $G$. Furthermore if $H=\left\langle y, G_{2}\right\rangle$ and $G=\langle x, y\rangle$ then.

$$
G_{i}=\left\langle U(i, 0), G_{i+1}\right\rangle
$$

for $i=r, \ldots, c$.

THEOREM 4.2. Let $G$ be the group described in Theorem 4.1. Suppose that

$$
U(r, 1)=\prod_{\nu=l}^{c} U(\nu, 0)^{b_{1}(\nu)}
$$

where $l \geq r+1$ and $b_{1}(l) \neq 0$. Suppose also that for $t \geq 2$

$$
U(r, t)=\prod_{\nu=r+1}^{c} U(\nu, 0)^{b_{t}(\nu)} .
$$

Then for $\nu=r+1, \ldots, c-1$,

$$
b_{t}(\nu)=\sum_{\left(x_{1}, \ldots, x_{t}\right)} b_{1}\left(x_{1}\right) \cdots b_{1}\left(x_{t}\right)
$$


where the summation is on those $\left(x_{1}, \ldots, x_{t}\right)$ where $x_{1}+\cdots+x_{t}=\nu+(t-1) r$.

The assumption, in Theorem 4.2, that $b_{1}(l) \neq 0$ for some $l \leq c$ is made for the sake of convenience. If $U(r, 1)=1$ then $b_{t}(\nu)=0$ for $t \geq 2$ and $\nu \leq c-1$. It should be noted that $b_{1}(l), \ldots, b_{1}(c)$ and $b_{t}(c)$ for $t=2, \ldots, r-2$ are the independent parameters here. The $b_{t}(\nu)$ where $t \geq 2$ and $\nu \leq c-1$ are functions of the $b_{1}(\nu)$.

THEOREM 4.3. Let $G$ be a group that satisfies the hypothesis of Theorem 4.1. Then $G$ has one of two forms. First, we may have $G_{i}=\left\langle U(i, 0), U(i, 1), G_{i+1}\right\rangle$ for $i=3, \ldots, r-1$ and

$$
U(4,2)=\prod_{i=k}^{c} U(i, 0)^{A(i)} U(i, 1)^{B(i)}
$$

where $k \geq 5$ and $B(i)=0$ for $i \geq r$. In addition for $\nu=r+1, \ldots, c$

$$
A(\nu-r+4)+\sum_{(i, j)} B(i) b_{1}(j)=b_{2}(\nu)
$$

where the summation is on those $(i, j)$ with $i+j=\nu+4$. Second, we may have $G_{i}=\left\langle U(i, 0), U(i, i-2), G_{i+1}\right\rangle$ for $i=3, \ldots, r-1$ and

$$
U(4,1)=\prod_{i=k}^{c} U(i, 0)^{A(i)} U(i, i-2)^{B(i)}
$$

where $k \geq 5$ and $B(i)=0$ for $i \geq r$. In addition for $\nu=r+1, \ldots, c$

$$
A(\nu-r+4)+\sum_{(i, j)} B(i) b_{i-2}(j)=b_{1}(\nu)
$$

where the summation is on these $(i, j)$ with $i+j=\nu+4$.

The summations in Theorem 4.3 are made with the usual conventions; e.g., the parameters $A(i)$ are zero unless $i$ is in the range $k \leq i \leq c$.

THEOREM 4.4. Suppose that $G$ satisfies the hypothesis of Theorem 4.3. Then $G=\langle x, y\rangle$ where $H=\left\langle y, G_{2}\right\rangle$ and, except in one case, $\left\langle x, G_{2}\right\rangle$ is a characteristic subgroup of index $p$ in $G$. In the exceptional case all of the following three conditions must hold: (i) $2 l-r \geq c$, (ii) $b_{3}(c)=\cdots=b_{r-2}(c)=0$, (iii) $(r-3) b_{2}(c)=$ $(l-2) h(b,(l))^{2}$ where $h=1$ if $2 l-r=c$ and $h=0$ if $2 l-r>c$.

The characteristic subgroup $\left\langle x, G_{2}\right\rangle$ of Theorem 4.4 is obtained by normalizing a parameter: When $2 l-r \leq c-1$ we choose $\lambda$ so that $b_{1}(2 l-r)=0$. When $2 l-r \geq c$ we set $b_{2}^{\prime}=(r-3) b_{2}(c)-h(l-2)\left(b_{1}(l)\right)^{2}$ and $b_{\nu}^{\prime}(c)=b_{\nu}(c)$ for $\nu=3, \ldots, r-2$. We suppose there is a $q$ such that $b_{q}^{\prime}(c) \neq 0$ and $b_{\nu}^{\prime}(c)=0$ for $\nu=q+1, \ldots, r-2$. We then choose $x$ so that $b_{q-1}(c)=0$. In both cases, if $X$ is the set of $x$ such that the appropriate parameter is zero then $\left\langle x, G_{2}\right\rangle$ is a characteristic subgroup of index $p$ in $G$.

Further information on the normalization of the parameters mentioned above is given in Lemmas 4.8 through 4.11 .

The isomorphism problem for those groups of Theorem 4.3 which are not one of the exceptional groups of Theorem 4.4 is quite simple. Any isomorphism $v$ between such groups must be of the form $\bar{x}^{v}=x^{\alpha}, \bar{y}^{v}=y^{\delta}$ and, consequently, 
$\bar{U}(i, j)^{v}=U(i, j)^{\varepsilon(i, j)}$ where $\varepsilon(i, j)=\alpha^{i-j-1} \delta^{j+1}$. Applying these results to the defining relations of the groups we obtain the isomorphism equations.

The isomorphism problem for the exceptional groups is also fairly straightforward. If $G$ is a group of the first type mentioned in Theorem 4.3 then, upon dividing through by $G_{r}$, we obtain a group of the type considered in Theorem 3.1. Thus, "most of the time", $G$ will have a pair of characteristic subgroups of index $p$. Furthermore if it does not-a case that occurs only when the parameters take on a very particular set of values-it will still have one characteristic subgroup and the isomorphism equations can be obtained from Lemma 2.1.

If $G$ is a group of the second type mentioned in Theorem 4.3 then $G / G_{r}$ is a group of the kind considered in Theorem 3.2. Since $G$ has a characteristic subgroup $H=\left\langle y, G_{2}\right\rangle$ of index $p$ and since, by Theorem 3.2, the isomorphisms of $G / G_{r}$ have a given form the isomorphisms of $G$ will be of the form $\bar{x}^{v}=x^{\alpha}, \bar{y}^{v}=y^{\delta}$. This, as usual, gives us the classification.

We now turn to the proofs of these theorems.

LEMMA 4.1. Suppose that $\left|G_{i} / G_{i+1}\right|=p$ for $i=r, \ldots, c$ and $r<c$. Let $H$ be the largest subgroup of $G$ such that $\left[H, G_{r}\right] \leq G_{r+2}$. Then $H$ is a characteristic subgroup of index $p$ in $G$.

ProOf. First, we may assume that $G_{r+2}=\{1\}$. Then $G_{r}$ is a subgroup of order $p^{2}$. Second

$$
G / C_{G}\left(G_{r}\right) \cong Q
$$

where $Q$ is some subgroup of the automorphism group of $G_{r}$. Since $\mid$ Aut $G_{r} \mid=$ $\left(p^{2}-1\right)\left(p^{2}-p\right)$ it follows that $\left|G / C_{G}\left(G_{r}\right)\right| \leq p$. Thus we take $H=C_{G}\left(G_{r}\right)$.

LEMMA 4.2. Suppose that $\left|G_{i} / G_{i+1}\right|=p$ for $i=r, r+1, \ldots, c, r<c$ and $G=\langle x, y\rangle$ with $\left[G_{r}, y\right] \leq G_{r+2}$. Then

$$
G_{i}=\left\langle u(i, 0), G_{i+1}\right\rangle, \quad i=r, \ldots, c .
$$

In addition $u(i, \nu) \in G_{i+1}$ for $i \geq r$ and $\nu \geq 1$.

Proof. Consider first those $u(i, \nu)$ where $i \geq r+1$ and $\nu \geq 1$. If we set $i=r+1+t$ we have

$$
\begin{aligned}
u(i, \nu) & =[u, x, \underbrace{\ldots}_{r-1+t-\nu}, x, y, \underbrace{\ldots}_{\nu}, y] \\
& =[u, x, \underbrace{\ldots}_{r-1-\nu}, x, y, \underbrace{\ldots}_{\nu-1}, y, y, x, \underbrace{\ldots}_{t}, x] \\
& =[u(r, \nu-1), y, x, \underbrace{\ldots}_{t}, x] \in G_{r+2+t}=G_{i+1} .
\end{aligned}
$$

Thus $G_{i}=\left\langle u(i, 0), u(i, 1), \ldots, u(i, i-2), G_{i+1}\right\rangle=\left\langle u(i, 0), G_{i+1}\right\rangle$ when $i \geq r+1$.

When $i=r$ we have $G_{r}=\left\langle u(r, 0), u(r, 1), \ldots, u(r, r-2), G_{r+1}\right\rangle$ and $\left|G_{r} / G_{r+1}\right|=$ $p$. Thus $G_{r}=\left\langle u(r, \nu), G_{r+1}\right\rangle$ for some $\nu, 0 \leq \nu \leq r-2$. We shall show that we must have $\nu=0$.

Consider a nontrivial relation of the form

$$
u(r, 0)^{a(0)} u(r, 1)^{a(1)} \cdots u(r, r-2)^{a(r-2)} \equiv 1 \bmod G_{r+1} .
$$


If we take the commutator of this with $x$ and apply the result of the previous paragraph we have

$$
u(r+1,0)^{a(0)} \equiv 1 \bmod G_{r+2} .
$$

Since $G_{r+1}=\left\langle u(r+1,0), G_{r+1}\right\rangle$ and $\left|G_{r+1} / G_{r+2}\right|=p$ it follows that $a(0)=0$.

So suppose that $G_{r}=\left\langle u(r, \nu), G_{r+1}\right\rangle$ for some $\nu \geq 1$. We would then have

$$
u(r, 0) \equiv u(r, \nu)^{a(\nu)} G_{r+1} .
$$

This is a nontrivial relation of the form (1) with $a(0) \neq 0$, which is a contradiction. Consequently, $G_{r}=\left\langle u(r, 0), G_{r+1}\right\rangle$.

Finally, $u(r, \nu) \in G_{r+1}$ for $\nu \geq 1$. If this were not the case we would have

$$
u(r, \nu)=u(r, 0)^{a} G_{r+1}
$$

where $a \neq 0$. Thus, again, we have a nontrivial relation of the form (1) with $a(0) \neq 0$.

LEMma 4.3. Let $U(i, j)$ be defined as in the introduction. Then

$$
G_{i}=\left\langle U(i, 0), G_{i+1}\right\rangle
$$

for $i=r, \ldots, c$.

PROOF. This follows from Lemma 4.2 and the fact that

$$
U(i, j) \equiv u(i, j) \bmod G_{i+1} .
$$

The above results give us Theorem 4.1.

LEMma 4.4. Suppose that

$$
U(r, 1)=\prod_{\nu \geq l} U(\nu, 0)^{b_{2}(\nu)}
$$

where $l \geq r+1$ and $b_{1}(l) \neq 0$. Set

$$
U(r, t)=\prod_{\nu \geq r+1} U(\nu, 0)^{b_{t}(\nu)} .
$$

Then for $\nu=r+1, \ldots, c-1$

$$
b_{t}(\nu)=\sum_{\left(x_{1}, \ldots, x_{t}\right)} b_{1}\left(x_{1}\right) \cdots b_{1}\left(x_{t}\right)
$$

the summation being on those $\left(x_{1}, \ldots, x_{t}\right)$ where $x_{1}+\cdots+x_{t}=\nu+(t-1) r$. Furthermore for $i \geq r+1$ and $t \geq 1$

$$
U(i, t)=\prod_{\nu \geq i+(l-r) t} U(\nu, 0)^{b_{t}(\nu-i+r)} .
$$

PROOF. By Lemma 2.3 and (3)

$$
U(r+1, t)=\prod_{\nu \geq r+2} U(\nu, 0)^{b_{t}(\nu-1)} .
$$

That is for $t=1, \ldots, r-3$

$$
U(r+1, t+1)=\prod_{\nu \geq r+2} U(\nu, 0)^{b_{t+1}(\nu-1)} .
$$


Next, from (2) and Lemma 2.3

$$
U(i, 1)=\prod_{\nu \geq l+i-r} U(\nu, 0)^{b_{1}(\nu-i+r)}
$$

for $i \geq 1$.

Consequently, by Lemma 2.3 and (3)

$$
U(r+1, t+1)=\prod_{\nu \geq r+1} U(\nu+1,1)^{b_{t}(\nu)}=\prod_{\nu \geq l+2} U(\nu, 0)^{S(\nu)}
$$

where

$$
S(\nu)=\sum_{i=r+2}^{\nu-l+r} b_{1}(\nu-i+r) b_{t}(i-1)
$$

Comparing exponents in (5) and (6) we obtain

$$
b_{t+1}(\nu)=\sum_{i=r+2}^{\nu-l+r+1} b_{1}(\nu-i+r+1) b_{t}(i-1)
$$

for $t=1, \ldots, r-3$ and $\nu=r+1, \ldots, c-1$. This equation can be written as

$$
b_{t+1}(\nu)=\sum_{x_{1}+x_{2}=\nu+r} b_{1}\left(x_{1}\right) b_{1}\left(x_{2}\right)
$$

for $t \geq 1$ and $\nu=r+1, \ldots, c-1$. Thus, by the recursive properties of $(7)$

$$
b_{t}(\nu)=\sum_{x_{1}+\cdots+x_{t}=\nu+(t-1) r} b_{1}\left(x_{1}\right) \cdots b_{1}\left(x_{t}\right)
$$

for $t \geq 1$ and $\nu \leq c-1$.

Next, since $b_{1}(x)=0$ for $x<l$ we must have $\nu \geq r+(l-r) t$ to have nonzero terms in the defining sum for $b_{t}(\nu)$. Consequently

$$
U(r, t)=\prod_{\nu \geq r+(l-r) t}^{c-1} U(\nu, 0)^{b_{t}(\nu)} \cdot U(c, 0)^{b_{t}(c)}
$$

If we apply Lemma 2.3 here we obtain (4).

Theorem 4.2 now follows from Lemma 4.4 .

Lemmas 4.5 through 4.11 deal with the proof of Theorem 4.4. In what follows we assume that $G=\langle x, y\rangle$ is isomorphic to $\bar{G}=\langle\bar{x}, \bar{y}\rangle$ under $v$ where $\bar{x}^{v}=x^{\alpha} y^{\beta}$ and $\bar{y}^{v}=y^{\delta}$. We also use the fact that since $\left[G_{r}, H\right]=G_{l}$ the integer $l$ is an isomorphism invariant. Thus we have, in $\bar{G}$,

$$
\bar{U}(r, 1)=\prod_{\nu \geq l} \bar{U}(\nu, 0)^{\bar{b}_{1}(\nu)}
$$

We shall be working modulo $G_{2 l-r+1}$. The following list of relations will be needed for our computations. 
LEMMA 4.5. We have

(a) For $i \geq r$,

$$
U(i, 1)=\prod_{\nu=l+i-r}^{c} U(\nu, 0)^{b_{1}(\nu+r-1)} .
$$

(b) If $2 l-r \leq c-1$, then

$$
U(r, 2) \equiv U(2 l-r, 0)^{b_{2}(2 l-r)} G_{2 l-r+1} .
$$

If $2 l-r \geq c$, then

$$
U(r, 2)=U(c, 0)^{b_{2}(c)}
$$

(c) If $t \geq 3$, then

$$
U(r, t) \equiv U(c, 0)^{b_{t}(c)} G_{2 l-r+1} .
$$

(d) If $i \geq r+1$ and $t \geq 2$, then

$$
U(i, t) \equiv 1 \quad \bmod G_{2 l-r+1} .
$$

Proof. Part (a) follows from the $t=1$ case of (8). If we take $t=2$ in (8) we obtain, for $2 l-r \leq c-1$

$$
U(r, 2) \equiv U(2 l-r, 0)^{b_{2}(2 l-r)} U(c, 0)^{b_{2}(c)} \bmod G_{2 l-r+1} .
$$

Since $c \geq 2 l-r+1$ this gives us the first part of (b). The second part of (b) and the balance of Lemma 4.5 follows from similar considerations.

Lemma 4.6. Let $s=1$ when $2 l-r \leq c-1$ and $s=0$ for $2 l-r \geq c$. Set

$$
A(s)=s b_{2}(2 l-r) f(r, 1, r, 2)
$$

and

$$
B(s)=(1-s) \sum_{t=2}^{r-2} f(r, 1, r, t) b_{t}(c) .
$$

Then, modulo $G_{2 l-r+1}$,

$$
\bar{U}(r, 1)^{v} \equiv\left(\prod_{\nu \geq l} U(\nu, 0)^{b_{1}(\nu)}\right)^{\alpha^{r-2} \delta^{2}} U(2 l-r, 0)^{A(s)} U(c, 0)^{B(s)} .
$$

PROOF. By Lemma 2.4 and part (d) of Lemma 4.5,

$$
\bar{U}(r, 1)^{v} \equiv \prod_{i \geq r} U(i, 1)^{f(r, 1, i, 1)} \prod_{t \geq 2} U(r, t)^{f(r, 1, r, t)} .
$$

By Lemma $2.2, f(r, 1, i, 1)=\delta(i, r) \alpha^{n-2} \delta^{2}$. Thus the first product in the line above is equal to $U(r, 1)^{f(r, 1, r, 1)}$ which, in turn, is equal to the product of Lemma 4.6. The balance of the result follows from Lemma 4.5.

LEMMA 4.7. We have

$\prod_{\nu \geq l}\left(\bar{U}(\nu, 0)^{v}\right) \equiv \prod_{\nu \geq l} U(\nu, 0)^{\bar{b}_{1}(\nu) \alpha^{\nu-1} \delta} \cdot U(2 l-r, 0)^{b_{1}(l) \bar{b}_{1}(l) f(l, 0, l, 1)} \bmod G_{2 l-r+1}$.

ProOF. Set

$$
S(\nu, t)=\sum_{i=l}^{\nu} \bar{b}_{1}(i) f(i, 0, \nu, t)
$$


Then, by the usual arguments,

$$
\begin{aligned}
\prod_{i \geq l}\left(\bar{U}(i, 0)^{v}\right)^{\bar{b}_{1}(i)} & =\prod_{\nu \geq l} \prod_{t \geq 0} U(\nu, t)^{S(\nu, t)} \\
& =\prod_{\nu \geq l} U(\nu, 0)^{S(\nu, 0)} \prod_{\nu \geq l} U(\nu, 1)^{S(\nu, 1)}
\end{aligned}
$$

In addition

$$
S(\nu, 0)=\sum_{i=l}^{\nu} \bar{b}_{1}(i) f(i, 0, \nu, 0)=\bar{b}_{1}(\nu) f(\nu, 0, \nu, 0)
$$

and

$$
S(l, 1)=\bar{b}_{1}(l) f(l, 0, l, 1) .
$$

These results give us Lemma 4.7.

LEMMA 4.8. Suppose that $2 l-r \leq c-1$. Then

(a) For $\nu=l, \ldots, 2 l-r-1$

$$
b_{1}(\nu) \alpha^{r-2} \delta^{2}=\bar{b}_{1}(\nu) \alpha^{\nu-1} \delta
$$

(b) For $\nu=2 l-r$

$$
b_{1}(2 l-r) \alpha^{r-2} \delta^{2}=\bar{b}_{1}(2 l-r) \alpha^{2 l-r-1} \delta+(l-r+1)\left(b_{1}(l)\right)^{2} \alpha^{r-3} \delta^{2} \beta .
$$

PROOF. Part (a) is obtained by comparing exponents in the products of Lemmas 4.6 and 4.7 .

As for part (b), Lemmas 4.6 and 4.7 give us, when $\nu=2 l-r$

$$
\begin{aligned}
b_{1}(2 l & -r) \alpha^{r-2} \delta^{2}+b_{2}(2 l-r) f(r, 1, r, 2) \\
& =\bar{b}_{1}(2 l-r) \alpha^{2 l-r-1} \delta+b_{1}(l) \bar{b}_{1}(l) f(l, 0, l, 1) .
\end{aligned}
$$

Then, by Lemma $4.4, b_{2}(2 l-r)=b_{1}(l) b_{1}(l)$. By Lemma $2.2, f(r, 1, r, 2)=$ $(r-3) \alpha^{r-3} \beta \delta^{2}$ and $f(l, 0, l, 1)=(l-2) \alpha^{l-2} \beta \delta$. Finally, by part (a) of Lemma $4.8, b_{1}(l) \alpha^{r-2} \delta^{2}=\bar{b}(l) \alpha^{l-1} \delta$. These results give us part (b).

Lemma 4.8 gives us a second characteristic group of index $p$ when $2 l-r \leq c-1$.

We shall now assume that $2 l-r \geq c$. Then, by (8) for $t=2, \ldots, r-2$

$$
U(r, t)=U(c, 0)^{b_{t}(c)} \text {. }
$$

LEMMA 4.9. Suppose $2 l-r \geq c$ and, for $t \geq 2$,

$$
\bar{U}(r, t)=\bar{U}(c, 0)^{\bar{b}_{t}(c)} \text {. }
$$

Then, for $t=2, \ldots, r-2$

$$
\alpha^{c-1} \delta \bar{b}_{t}(c)=\sum_{\nu=t}^{r-2} f(r, t, r, \nu) b_{\nu}(c) .
$$

ProOF. By Lemma 2.1

$$
\bar{U}(r, t)^{v}=\prod_{m \geq r} \prod_{\nu=t}^{m-2} U(m, \nu)^{f(r, t, m, \nu)} .
$$


Next, equation (9) implies that $U(i, \nu)=1$ when $i \geq r+1$ and $\nu \geq 1$. Thus

$$
\bar{U}(r, t)=\prod_{\nu=t}^{r-2} U(r, \nu)^{f(r, t, r, \nu)}=\prod_{\nu=t}^{r-2} U(c, 0)^{b_{\nu}(c) f(r, t, r, \nu)} .
$$

Similarly

$$
\begin{aligned}
\bar{U}(c, 0)^{v} & =\prod_{m \geq c} \prod_{q=0}^{m-2} U(m, q)^{f(c, 0, m, q)} \\
& =U(c, 0)^{f(c, 0, c, q)}=U(c, 0) \alpha^{c-1} \delta .
\end{aligned}
$$

LEMMA 4.10. Suppose that $b_{\nu}(c)=0$ for $\nu=q+1, \ldots, r-2$. Then $\bar{b}_{\nu}(c)=0$ for $\nu=q+1, \ldots, r-2$. In addition

$$
\alpha^{c-1} \delta \bar{b}_{q}(c)=\alpha^{r-q-1} \delta^{q+1} b_{q}(c)
$$

and

$$
\alpha^{c-1} \delta \bar{b}_{q-1}(c)=\alpha^{r-q} \delta^{q} b_{q-1}(c)+(r-q-1) \alpha^{r-q-1} \delta^{q+1} \beta b_{q}(c) .
$$

Proof. This follows directly from Lemma 4.9.

If for some $q \geq 3$ we have $b_{q}(c) \neq 0$ then, once again we have a second characteristic of index $p$ in $G$. It is obtained by the normalization $b_{q-1}(c)=0$.

To continue, we suppose that $b_{3}(c)=\cdots=b_{r-2}(c)=0$ in what follows.

LEMMA 4.11. Suppose that $2 l-r \geq c$ and

$$
b_{3}(c)=b_{4}(c)=\cdots=b_{r-2}(c)=0 .
$$

Set

$$
h= \begin{cases}1 & \text { if } 2 l-r=c, \\ 0 & \text { if } 2 l-r>c .\end{cases}
$$

We then have

$$
\alpha^{c-1} \delta \bar{b}_{2}(c)=\alpha^{r-3} \delta^{3} b_{2}(c)
$$

and

$$
\alpha^{c-1} \delta \bar{b}_{1}(c)-\left[(r-3) b_{2}(c)-h(l-2)\left(b_{1}(l)\right)^{2}\right] \alpha^{r-3} \delta^{2} \beta=\alpha^{r-2} \delta^{2} b_{2}(c) .
$$

PROOF. The first equation is a consequence of Lemma 4.10. Next, by Lemmas 4.7 and 4.6 ,

$$
\bar{b}_{1}(c) \alpha^{c-1} \delta+h b_{1}(l) \bar{b}_{1}(l) f(l, 0, l, 1)=b_{1}(c) \alpha^{r-2} \delta^{2}+f(r, 1, r, 2) b_{2}(c) .
$$

This gives us the second.

We now turn to the proof of Theorem 4.3. This is given in Lemmas 4.12 through 4.19 .

LEMMA 4.12. Suppose that

$$
\left|G_{i} / G_{i+1}\right|=p^{2}, \quad i=q, \ldots, r-1 .
$$

Then, for $i=q, \ldots, r-1$, the basis of $G_{i}$ modulo $G_{i+1}$ has one of two forms:

(a) In the first case there is a $\lambda$, with $1 \leq \lambda \leq q-2$ such that

$$
G_{i}=\left\langle u(i, 0), u(i, \lambda), G_{i+1}\right\rangle, \quad i=q, \ldots, r-1 .
$$


In addition there are integers $b(i, t)$ such that

$$
u(i, t)=u(i, \nu)^{b(i, t)} \bmod G_{i+1}, \quad t=1, \ldots, i-2 .
$$

(b) In the second case we have

$$
G_{i}=\left\langle u(i, 0), u(i, i-2), G_{i+1}\right\rangle, \quad i=q, \ldots, r-1 .
$$

In addition

$$
u(i, t) \equiv 1 \quad \bmod G_{i+1}, \quad t=1, \ldots, i-3 .
$$

There are several steps to the proof. We start with

LEMMA 4.13. Suppose that $\left|G_{i} / G_{i+1}\right|=p^{2}$ for $i=q, \ldots, r-1$ and $y$ is chosen so that $\left[G_{r}, y\right] \leq G_{r+2}$. Suppose that

$$
u(i, 0)^{a(0)} u(i, 1)^{a(1)} \cdots u(i, i-2)^{a(t-2)} \equiv 1 \bmod G_{i+1} .
$$

Then $a(0)=0$. In particular $u(i, 0) \notin G_{i+1}$.

ProOF. The proof is similar to the proof of (1) in the proof of Lemma 4.2.

LEMMA 4.14. There is a with $1 \leq \nu \leq q-2$ such that

$$
G_{q}=\left\langle u(q, 0), u(q, \nu), G_{q+1}\right\rangle .
$$

ProOF. Consider the vector space of dimension 2

$$
G_{q} / G_{q+1}=\left\langle u(q, 0) G_{q+1}, u(q, 1) G_{q+1}, \ldots, u(q, q-2) G_{q+1}\right\rangle .
$$

By Lemma 4.13, $u(q, 0) G_{q+1}$ is a basis element. Next, since the dimension is 2 , there is a $\nu \geq 1$ such that $u(q, \nu) G_{q+1} \neq G_{q+1}$. Finally, by Lemma $4.13, u(q, 0) G_{q+1}$ and $u(q, \nu) G_{q+1}$ are linearly independent.

LEMMA 4.15. Suppose that

$$
G_{q}=\left\langle u(q, 0), u(q, \lambda), G_{q+1}\right\rangle
$$

where $\lambda \leq q-3$. Then

$$
G_{i}=\left\langle u(i, 0), u(i, \lambda), G_{i+1}\right\rangle
$$

for $i=q, \ldots, r-1$. In addition, for $t \neq 0$

$$
u(i, t) \equiv u(i, \lambda)^{b(i, t)} \bmod G_{i+1} .
$$

Proof. By the proof of Lemma 4.14 , there is a $b_{t}$ such that

$$
u(q, t) \equiv u(q, \lambda)^{b_{t}} G_{q+1}
$$

for $t=1,2, \ldots, q-2$. Taking the commutator of this by $x$ we get

$$
u(q+1, t)=u(q+1, \lambda)^{b_{t}} \bmod G_{q+2}
$$

for $t \leq q-2$.

Next set $t=q-2$ in (1) and take the commutator of the result by $y$ to get, by

(3) $u(q+1, q-1) \equiv u(q+1, \lambda+1)^{b_{q-2}} \equiv u(q+1, \lambda)^{b_{q-2} b_{\lambda+1}} \quad \bmod G_{q+2}$.

Thus by (2) and (3),

$$
G_{q+1}=\left\langle u(q+1,0), u(q+1, \lambda), G_{q+2}\right\rangle .
$$

The proof now proceeds by induction. 
LEMMA 4.16. Suppose that $G_{q}=\left\langle u(q, 0), u(q, q-2), G_{q+1}\right\rangle$. Then $G_{i}=$ $\left\langle u(i, 0), u(i, q-2), G_{i+1}\right\rangle$ for $i=q, \ldots, r-1$ or $G_{i}=\left\langle u(i, 0), u(i, i-2), G_{i+1}\right\rangle$ for $i=q, \ldots, r-1$.

ProOF. We have

$$
u(q, t) \equiv u(q, q-2)^{b_{t}} \quad \bmod G_{q+1} .
$$

Thus, for $t=1, \ldots, q-2$

$$
u(q+1, t) \equiv u(q+1, q-2)^{b_{t}} \bmod G_{q+1} .
$$

Consequently

$$
g_{q+1}=\left\langle u(q, 0), u(q+1, q-2), u(q+1, q-1), G_{q+2}\right\rangle .
$$

This implies that there is a nontrivial relation of the form

$$
u(q+1, q-2)^{a} u(q+1, q-1)^{b} \equiv 1 \bmod G_{q+2} .
$$

Consider the case where $b \neq 0$. We then have

$$
u(q+1, q-1) \equiv u(q+1, q-2)^{\bar{a}} \bmod G_{q+2}
$$

for some number $\bar{a}$. The proof in this case now follows the proof of Lemma 4.15.

Suppose next that, in (5), $b=0$. We then have

$$
u(q+1, q-2) \equiv 1 \bmod G_{q+2} \text {. }
$$

Thus

$$
G_{q+1}=\left\langle u(q+1,0), u(q+1, q-1), G_{q+1}\right\rangle .
$$

So suppose, inductively, that

$$
G_{i}=\left\langle u(i, 0), u(i, i-2), G_{i+1}\right\rangle
$$

with

$$
u(i, t) \equiv 1 \bmod G_{i+1}
$$

for $t=1, \ldots, i-3$. We then have

$$
u(i+1, t)=[u(i, t), x] \equiv 1 \bmod G_{i+2}
$$

for $t=1, \ldots, i-3$ and

$$
u(i+1, i-2)=[u(i, i-3), y] \equiv 1 \bmod G_{i+2} .
$$

That is, the inductive step follows.

This completes the proof of Lemma 4.12.

We now return to our assumption that $\left|G_{2} / G_{3}\right|=p,\left|G_{i} / G_{i+1}\right|=p^{2}, i=$ $3, \ldots, r-1$ and $\left|G_{i} / G_{i+1}\right|=p$ for $i=r, \ldots, c$. By Theorem 2 we have three possible relations modulo $G_{5}$ :

$$
u(4,2) \equiv 1, \quad u(4,1) \equiv 1, \quad u(4,0)^{-d} u(4,2) \equiv 1 .
$$

By (1) of the proof of Lemma 4.1, the last is impossible. Thus there are two relations to consider. The case where $u(4,2) \equiv 1$ corresponds to part (a) of Lemma 4.12 ; part (b) corresponds to $u(4,1) \equiv 1$.

We now turn to the consistency problem. We have one defining relation

$$
U(r, t)=\prod_{\nu=r+1}^{c} U(\nu, 0)^{b_{t}(\nu)}
$$

for $t=1, \ldots, r-2$ and will introduce another. The relations must be consistent. 
LEMMA 4.17. Suppose that

$$
G_{i}=\left\langle U(i, 0), U(i, 1), G_{i+1}\right\rangle, \quad i=3, \ldots, r-1,
$$

and

$$
G_{i}=\left\langle U(i, 0), G_{i+1}\right\rangle, \quad i=r, \ldots, c .
$$

Suppose that

$$
U(4,2)=\prod_{i=k}^{c} U(i, 0)^{A(i)} U(i, 1)^{B(i)}
$$

where $k \geq 5,(A(k), B(k)) \neq(0,0)$, and $B(i)=0$ for $i \geq r$. Then (6) and (7) are consistent and only if the following two sets of conditions both hold.

(a) For $\nu=r+1, \ldots, c$,

$$
A(\nu-r+4)+\sum_{x_{1}+x_{2}=\nu+4} B\left(x_{1}\right) b_{1}\left(x_{2}\right)=b_{2}(\nu) .
$$

(b) For $\nu=r+1, \ldots, c$ and $t \geq 1$

$$
\sum_{x_{1}+x_{2}=\nu+4}\left(b_{t}\left(x_{1}\right) A\left(x_{2}\right)+b_{t+1}\left(x_{1}\right) B\left(x_{2}\right)\right)=b_{t+2}(\nu) .
$$

PROOF. If we apply Lemma 2.3 to (7) we have, for $t \geq 0$,

$$
U(r, t+2)=\prod_{\nu \geq r+k-4} U(\nu, t)^{A(\nu-r+4)} \prod_{\nu \geq k+r-4} U(\nu, t+1)^{B(\nu-r+4)} .
$$

Now, for $t \geq 0$

$$
U(r, t+2)=\prod_{\nu \geq r+1} U(\nu, 0)
$$

In addition

$$
\prod_{\nu \geq k+r-4} U(\nu, t+1)^{B(\nu-r+4)}=\prod_{\nu \geq k+r-3} U(\nu, 0)^{S_{t+1}(\nu)}
$$

where

$$
S_{t+1}(\nu)=\sum_{x_{1}+x_{2}=\nu+4} b_{t+1}\left(x_{1}\right) B\left(x_{2}\right)
$$

Lemma 4.17 follows from these results. If we take $t=0$ we obtain part (a); part (b) follows from the $t \geq 1$ case.

LEMMA 4.18. If the equations in part (a) of Lemma 4.17 hold then so do those in part (b).

PROOF. This follows from the identity

$$
b_{t+1}(\nu)=\sum_{x_{1}+x_{2}=\nu+r} b_{1}\left(x_{1}\right) b_{t}\left(x_{2}\right),
$$

which appears in the proof of Lemma 4.4. 
LEMMA 4.19. Suppose that

$$
\begin{aligned}
G_{i} & =\left\langle U(i, 0), U(i, i-2), G_{i+1}\right\rangle, & & i=3, \ldots, r-1, \\
G i & =\left\langle U(i, 0), G_{i+1}\right\rangle, & & i=r, \ldots, c
\end{aligned}
$$

and

$$
U(4,1)=\prod_{i=k}^{c} U(i, 0)^{A(i)} \prod_{i=k}^{c} U(i, i-2)^{B(i)}
$$

where $k \geq 5,(A(k), B(k)) \neq(0,0)$ and $B(i)=0$ for $i \geq r$. Then

(a) For $\nu=r+1, \ldots, c$,

$$
A(\nu-r+4)+\sum_{x_{1}+x_{2}=\nu+4} b_{x_{2}-2}\left(x_{1}\right) B\left(x_{2}\right)=b_{1}(\nu) .
$$

(b) For $\nu=r+1, \ldots, c$ and $t \geq 1$,

$$
\sum_{x_{1}+x_{2}=\nu+4}\left(b_{t}\left(x_{1}\right) A\left(x_{2}\right)+b_{x_{2}-2+t}\left(x_{1}\right) B\left(x_{2}\right)\right)=b_{t+1}(\nu) .
$$

PROOF. By the customary arguments

$$
\begin{aligned}
U(r, 1+t) & =\prod_{\nu \geq k+r-4} U(\nu, t)^{A(\nu-r+4)} U(\nu, \nu-r+2+t)^{B(\nu-r+4)}, \\
U(r, 1+t) & =\prod_{\nu \geq r+1} U(\nu, 0)^{b_{t+1}(\nu)}
\end{aligned}
$$

and

$$
\prod_{\nu \geq k+r-4} U(\nu, \nu-r+2+t)^{B(\nu-r+4)}=\prod_{\nu \geq k+r-3} U(\nu, 0)^{T(\nu)}
$$

where

$$
T(\nu)=\sum_{x_{1}+x_{2}=\nu+4} b_{x_{2}-2+t}\left(x_{1}\right) B\left(x_{2}\right) .
$$

In addition if $t \geq 1$ then

$$
\prod_{\nu \geq k+r-4} U(\nu, t)^{A(\nu-r+4)}=\prod_{\nu \geq k+r-3} U(\nu, 0)^{S(\nu)}
$$

where

$$
S(\nu)=\sum_{x_{1}+x_{2}=\nu+4} b_{t}\left(x_{1}\right) A\left(x_{2}\right) .
$$

Lemma 4.18 follows from these results.

As in the previous case the validity of the equations in part (a) implies the validity of those in part (b).

ACKNOWLEDGEMENT. This is the author's dissertation at the University of California at Los Angeles. The author would like to express his gratitude to Professor Miech for his encouragement and inspiration. 


\section{BIBLIOGRAPHY}

1. B. Huppert, Endliche Gruppen. I, Die Grundlehren der Math. Wissenchaften, Band 134, Springer-Verlag, Berlin and New York.

2. R. Miech, The metabelian p-groups of maximal class, Trans. Amer. Math. Soc. 236 (1978), 93-119.

3. _ A metabelian, prime power classification problem, J. London Math. Soc. (2) 23 (1981), 68-84.

4. _ A commutator basis, Quart. J. Math. Oxford Ser. (2) 135 (1983), 357.

Department of Mathematics, KaO-Hsiung Teacher's College, Taiwan, RePUBLIC OF CHINA 